\title{
ON THE MODULI SPACE OF SUPERMINIMAL SURFACES IN SPHERES
}

\author{
LUIS FERNÁNDEZ
}

Received 25 December 2001

\begin{abstract}
Using a birational correspondence between the twistor space of $S^{2 n}$ and projective space, we describe, up to birational equivalence, the moduli space of superminimal surfaces in $S^{2 n}$ of degree $d$ as curves of degree $d$ in projective space satisfying a certain differential system. Using this approach, we show that the moduli space of linearly full maps is nonempty for sufficiently large degree and we show that the dimension of this moduli space for $n=3$ and genus 0 is greater than or equal to $2 d+9$. We also give a direct, simple proof of the connectedness of the moduli space of superminimal surfaces in $S^{2 n}$ of degree $d$.
\end{abstract}

2000 Mathematics Subject Classification: 53A10, 53C42.

1. Introduction. A superminimal surface in $S^{2 n}$ can be defined as a harmonic map $f: M_{g} \rightarrow S^{2 n}$, where $M_{g}$ is a Riemann surface of genus $g$, whose osculating plane (i.e., the subspace spanned by all the derivatives of $f$ ) is totally isotropic (i.e., the symmetric complex product of any two vectors in that subspace is zero). These surfaces admit holomorphic lifts

$$
\Phi: M_{g} \longrightarrow \mathscr{L}_{n}
$$

into the twistor space $\mathscr{L}_{n}=\mathrm{SO}(2 n+1) / \mathrm{SO}(n)$ of the $2 n$-sphere (see $\left.[1,6,12]\right)$. When $f$ is linearly full (i.e., not contained in a proper subsphere of $S^{2 n}$ ), $\Phi$ is given by the osculating plane, at each point, of the map $f$. The study of these harmonic maps becomes then translated into the study of their twistor lifts, where more powerful tools in the complex analytic category can be used.

For the particular case of maps into $S^{4}$ (this is $n=2$ ), one has the added advantage that $\mathscr{L}_{2} \simeq \mathbb{P}^{3}$, so one can characterize twistor lifts of superminimal maps as solutions in $\mathbb{P}^{3}$ of a simple differential equation. In [11], Loo found that the dimension of the moduli space of harmonic spheres of degree $d$ (this is of area $4 \pi d$ ) in $S^{4}$ is $2 d+4$, and in [13, 14, 15], Verdier described the structure of this moduli space in detail.

For higher dimensions, the problem becomes harder simply because $\mathscr{E}_{n}$ is a more complicated object. In [5], Bryant uses homogeneous spaces to obtain a differential system similar to (2.13), and in [9], Hano finds the same system 
using Clifford algebras. Unfortunately their methods do not help in the study of the moduli space since the degree of the maps is not under control.

In this paper, we show that it is possible to use the same strategy for higher dimensions as in $S^{4}$, namely to translate the problem of studying the moduli space of superminimal surfaces of a certain degree into the study of maps into projective space satisfying the differential system (2.13). Although $\mathscr{E}_{n}$ and $\mathbb{P}^{N_{n}}$ (where $N_{n}$ is the dimension of $\mathscr{E}_{n}$ ) are of course not isomorphic, they are birationally equivalent. We exploit this birational correspondence to induce a birational equivalence between the moduli space of twistor lifts of superminimal maps from $M_{g}$ to $S^{2 n}$ of degree $d$ and the moduli space of maps from $M_{g}$ to $\mathbb{P}^{N_{n}}$ of degree $d$ that satisfy the differential system (2.13) (Theorem 3.1).

Using this result, we show in Theorem 4.1 that there exist harmonic maps from $S^{2}$ to $S^{2 n}$ which are linearly full (i.e., not contained in any proper subsphere of $S^{2 n}$ ) and have arbitrarily large area. In other words, the subset of the aforementioned moduli space consisting of linearly full maps is not empty for degree sufficiently large. Previous existence proofs are much weaker; in [1], explicit minimal maps $S^{2} \rightarrow S^{2 n}$ of arbitrarily large area are constructed, but they only work for genus 0 and in [5,9], there is no mention about the area, only the ramification degree, and the area is the main invariant of these maps.

In Section 5, we give a simple, direct, and geometric proof of the connectedness of the moduli space of superminimal surfaces in $S^{2 n}$ with degree $d$. We believe that the same method can be used to find other topological properties of the moduli space such as its fundamental group for arbitrary genus, generalizing the results in [7].

Finally, it is an interesting open problem to find the dimension of the set of harmonic spheres in $S^{2 n}$. The main conjecture is that this dimension is $2 d+n^{2}$. The methods described in the paper reduce this problem to finding the dimension of a variety formed by the intersection of a large number of quadrics. In Section 6, we show that the dimension of the moduli space of harmonic maps from $S^{2}$ to $S^{6}$ (i.e., $n=3$ ) is greater than or equal to $2 d+9$ (Theorem 6.2). We believe that this result can be extended to arbitrary $n$ using the same method.

\section{Preliminaries}

2.1. The differential system. Let $\left\{e_{0}, e_{1}, \ldots, e_{n}, e_{n+1}, \ldots, e_{2 n}\right\}$ be a unitary basis of $\mathbb{C}^{2 n+1}$, and let $E_{0}=e_{0}, E_{k}=\left(e_{2 k-1}-i e_{2 k}\right) / \sqrt{2}$, and $\bar{E}_{i}=\left(e_{2 k-1}+\right.$ $\left.i e_{2 k}\right) / \sqrt{2}$, with $1 \leq k \leq n$. Then, for vectors $\vec{x}=\left(x_{0}, x_{1}, \ldots, x_{n}, y_{1}, \ldots, y_{n}\right)$ and $\vec{x}^{\prime}=\left(x_{0}^{\prime}, x_{1}^{\prime}, \ldots, x_{n}^{\prime}, y_{1}^{\prime}, \ldots, y_{n}^{\prime}\right)$ expressed in the basis $\left\{E_{0}, E_{1}, \ldots, E_{n}, \bar{E}_{1}, \ldots, \bar{E}_{n}\right\}$, the complex symmetric product is given by

$$
\left(\vec{x}, \vec{x}^{\prime}\right)=x_{0} x_{0}^{\prime}+\sum_{j=1}^{n} x_{j} y_{j}^{\prime}+\sum_{j=1}^{n} x_{j}^{\prime} y_{j} .
$$


Recall that $\mathscr{L}_{n}$, the twistor space of $S^{2 n}$, is the subset of the Grassmannian consisting of isotropic $n$-planes. Let $E$ denote the $n$-plane $E_{1} \wedge E_{2} \wedge \cdots \wedge E_{n}$, and let $U_{E}$ denote the Zariski open subset of $\mathscr{L}_{n}$ consisting of those $n$-planes whose orthogonal projection onto $E$ is one to one and onto. Every $n$-plane in $U_{E}$ can be written as the plane generated, in the basis $\left(E_{0}, E, \bar{E}\right)$, by the rows of a matrix of the form

$$
\left(\begin{array}{ccccccccc}
\alpha_{1} & \mid 1 & 0 & \cdots & 0 & -\frac{\alpha_{1}^{2}}{2} & -\frac{\alpha_{1} \alpha_{2}}{2}-\tau_{12} & \cdots & -\frac{\alpha_{1} \alpha_{n}}{2}-\tau_{1 n} \\
\alpha_{2} & \mid 0 & 1 & \cdots & 0 & -\frac{\alpha_{2} \alpha_{1}}{2}-\tau_{21} & -\frac{\alpha_{2}^{2}}{2} & \cdots & -\frac{\alpha_{2} \alpha_{n}}{2}-\tau_{2 n} \\
\vdots & \mid \vdots & \vdots & \ddots & \vdots & \vdots & \vdots & \ddots & \vdots \\
\alpha_{n} & \mid 0 & 0 & \cdots & 1 & -\frac{\alpha_{n} \alpha_{1}}{2}-\tau_{n 1} & -\frac{\alpha_{n} \alpha_{2}}{2}-\tau_{n 2} & \cdots & -\frac{\alpha_{n}^{2}}{2}
\end{array}\right),
$$

where $\alpha_{i}$ and $\tau_{j k}$ are complex numbers satisfying $\tau_{j k}=-\tau_{k j}$.

For ease of notation, let

$$
\alpha=\left(\begin{array}{c}
\alpha_{1} \\
\alpha_{2} \\
\vdots \\
\alpha_{n}
\end{array}\right), \quad T=\left(\begin{array}{cccc}
0 & \tau_{12} & \ldots & \tau_{1 n} \\
-\tau_{12} & 0 & \ldots & \tau_{2 n} \\
\vdots & \vdots & \ddots & \vdots \\
-\tau_{1 n} & -\tau_{2 n} & \ldots & 0
\end{array}\right),
$$

and let

$$
A=\frac{\alpha^{t} \alpha}{2}=\frac{1}{2}\left(\begin{array}{cccc}
\alpha_{1}^{2} & \alpha_{1} \alpha_{2} & \cdots & \alpha_{1} \alpha_{n} \\
\alpha_{2} \alpha_{1} & \alpha_{2}^{2} & \cdots & \alpha_{2} \alpha_{n} \\
\vdots & \vdots & \ddots & \vdots \\
\alpha_{n} \alpha_{1} & \alpha_{n} \alpha_{2} & \cdots & \alpha_{n}^{2}
\end{array}\right)
$$

where the superscript $t$ on the left indicates the transpose of the matrix. With this notation, the matrix (2.2) can be written as

$$
\left(\alpha, I_{n},(-T-A)\right) .
$$

Since all the entries in the last matrix are holomorphic functions of $\alpha$, T, we have the following lemma.

LEMmA 2.1. For each choice of base plane $E$, the map $\mathbb{C}^{N_{n}} \rightarrow U_{E} \subseteq \mathscr{L}_{n}$ given by

$$
\begin{aligned}
\left(\alpha_{1}, \ldots, \alpha_{n}, \tau_{12}, \ldots, \tau_{1 n}, \tau_{23}, \ldots, \tau_{n-1, n}\right) & \\
& \longrightarrow\left\{\text { plane spanned by the rows of }\left(\alpha, I_{n},(-T-A)\right)\right\}
\end{aligned}
$$

is biholomorphic and provides a local parametrization of $\mathscr{E}_{n}$. (Here, $N_{n}:=n(n+$ 1) $/ 2=\operatorname{dim}\left(\mathscr{E}_{n}\right)$.) 
A superminimal map $f: M_{g} \rightarrow S^{2 n}$ admits a holomorphic lift $\Phi: M_{g} \rightarrow \mathscr{L}_{n}$ which is horizontal for the fibration (see $[1,6,12]$ )

$$
\mathrm{SO}(2 n) / U(n) \hookrightarrow \mathscr{L}_{n} \stackrel{\pi}{\longrightarrow} S^{2 n} .
$$

Conversely, given a holomorphic, horizontal map $\Phi: M_{g} \rightarrow \mathscr{L}_{n}$, the map $f=$ $\Phi \circ \pi$ is superminimal. An explicit formulation of horizontality is as follows: regarding a vector $v \in T_{P} \mathscr{E}_{n}$ as an element of $\operatorname{Hom}\left(P, P^{\perp}\right), v$ is horizontal if and only if $v(P) \perp \bar{P}$ (see [12] for details), or equivalently,

$$
v \text { horizontal } \Longleftrightarrow(v(x), y)=0 \quad \forall x, y \in P .
$$

Given a holomorphic and horizontal map $\Phi: M_{g} \rightarrow \mathscr{L}_{n}$, choose a base plane $E$ so that the image of $\Phi$ is almost everywhere contained in $U_{E}$. Taking a local trivialization on $M_{g}$, we can write $\Phi$ as $\left(\alpha, I_{n},(-T-A)\right)$, where $\alpha_{i}$ and $\tau_{j k}$ are holomorphic functions defined locally on $M_{g}$ and $A=\alpha^{t} \alpha / 2$ as before. Then $d \Phi$ is a $\operatorname{Hom}\left(P, P^{\perp}\right)$-valued holomorphic form taking a vector ${ }^{t} x \cdot\left(\alpha, I_{n},(-T-\right.$ $A)) \in P$ to the projection on $P^{\perp}$ of ${ }^{t} x \cdot(d \alpha, 0,-d A-d T)$. Thus, for all $x, y \in \mathbb{C}^{n}$,

$$
\Phi \text { horizontal } \Longleftrightarrow\left(\operatorname{proj}_{P \perp}\left\{{ }^{t} x \cdot(d \alpha, 0,-d A-d T)\right\},{ }^{t} y \cdot\left(\alpha, I_{n},(-T-A)\right)\right)=0 .
$$

Now, since $P$ is totally isotropic,

$$
\left(\operatorname{proj}_{P}\left\{{ }^{t} x \cdot(d \alpha, 0,-d A-d T)\right\},{ }^{t} y \cdot\left(\alpha, I_{n},(-T-A)\right)\right)=0 \quad \forall x, y \in \mathbb{C}^{n} .
$$

Therefore,

Фhorizontal

$$
\Longleftrightarrow\left({ }^{t} x \cdot(d \alpha, 0,-d A-d T),{ }^{t} y \cdot\left(\alpha, I_{n},(-T-A)\right)\right)=0 \quad \forall x, y \in \mathbb{C}^{n} .
$$

In matrix notation this takes the form

$$
d \alpha^{t} \alpha-d T-d A=0
$$

Note that this equation is independent of the local trivialization of $M_{g}$ chosen. Computing $d A$ and simplifying, we obtain the following proposition.

Proposition 2.2. Let $f: M_{g} \hookrightarrow S^{2 n}$ be a superminimal map. Let $\Phi$ be the twistor lift of $f$. Choose a base plane $E$ so that the image of $\Phi$ lies in $U_{E}$ except for finitely many points of $M_{g}$. Use the parametrization given in Lemma 2.1. Then, except at the finite number of points of $M_{g}$ where they are not defined, the functions $\alpha_{i}, \tau_{j k}$ satisfy the equations

$$
\alpha_{j} d \alpha_{i}-\alpha_{i} d \alpha_{j}=2 d \tau_{i j}
$$


or, in matrix notation,

$$
d \alpha^{t} \alpha-\alpha d^{t} \alpha=2 d T
$$

In addition, $f$ is linearly full if and only if $W\left(\alpha^{\prime}\right) \neq \equiv 0$, where $W\left(\alpha^{\prime}\right)$ denotes the Wronskian of the functions $\left\{\alpha_{1}, \alpha_{2}, \ldots, \alpha_{n}\right\}$.

Proof. We only need to prove the last statement. The map $f$ is not linearly full if and only if there is an isotropic constant vector $v$ contained in the plane $\Phi(p)$ at every point $p \in M_{g}$ (i.e., a vector perpendicular to the image of $f$ ). Using the parametrization of Lemma 2.1, this occurs if and only if there exists a nonzero $\mathbb{C}^{n}$-valued function $b$ such that ${ }^{t} b \cdot\left(\alpha, I_{n},(-T-A)\right)$ is constant.

If there is such a vector $b$, differentiating and using $d(-T-A)=-{ }^{t} \alpha d \alpha$, we obtain

$$
{ }^{t} b^{\prime} \cdot\left(\alpha, I_{n},(-T-A)\right)+{ }^{t} b \cdot\left(\alpha^{\prime}, 0_{n},-{ }^{t} \alpha \alpha^{\prime}\right)=0 .
$$

This implies ${ }^{t} b^{\prime}=0$ and ${ }^{t} b \alpha^{\prime}=0$. Therefore, $W\left(\alpha^{\prime}\right) \equiv 0$. Conversely, if $W\left(\alpha^{\prime}\right) \equiv 0$, there must be a constant vector $b$ such that ${ }^{t} b \alpha^{\prime}=0$, which implies ${ }^{t} b \cdot\left(\alpha^{\prime}, 0_{n},-{ }^{t} \alpha \alpha^{\prime}\right)=0$, and therefore ${ }^{t} b \cdot\left(\alpha, I_{n},(-T-A)\right)$ is constant.

The previous lemma gives a local characterization of lifts of harmonic maps $f: M_{g} \rightarrow S^{2 n}$. In order to study the moduli space of such maps, we need to be able to characterize them globally. To this end, we extend the parametrization given in Lemma 2.1 to a birational map $b_{n}: \mathbb{P}^{N_{n}} \rightarrow \mathscr{L}_{n}$ given by

$$
\begin{aligned}
& {\left[1: \alpha_{1}: \cdots: \alpha_{n}: \tau_{12}: \cdots: \tau_{1 n}: \tau_{23}: \cdots: \tau_{n-1, n}\right]} \\
& \quad \stackrel{b_{n}}{\longrightarrow}\left\{\text { plane spanned by rows of }\left(\alpha, I_{n},(-T-A)\right)\right\} .
\end{aligned}
$$

(We omit the dependence of $b_{n}$ on $E$ to simplify the notation.) Then, given a holomorphic map $\Phi: M_{g} \rightarrow \mathscr{L}_{n}$, the composition $b_{n}^{-1} \circ \Phi$ will be a birational map from $M_{g}$ into $\mathbb{P}^{N_{n}}$. This map can be completed to obtain a bona fide map $\widetilde{\Phi}: M_{g} \rightarrow \mathbb{P}^{N_{n}}$. In addition, it will be convenient for future computations to embed $\mathscr{L}_{n}$ inside $\mathbb{P}^{M_{n}}$ via the Plücker embedding (here $M_{n}=\left(\begin{array}{c}2 n+1 \\ n\end{array}\right)-1$ ). We obtain Figure 2.1.

In terms of the homogeneous coordinates $\left[s: \alpha_{1}: \cdots: \alpha_{n}: \tau_{12}: \cdots: \tau_{n-1, n}\right]$, (2.13) takes the form

$$
d \alpha^{t} \alpha-\alpha d^{t} \alpha=2(s d T-T d s)
$$

and the condition $W\left(\alpha^{\prime}\right) \not \equiv 0$ turns into

$$
W\left(\left(\frac{\alpha}{s}\right)^{\prime}\right) \not \equiv 0
$$

The main result of this section is the following theorem. 


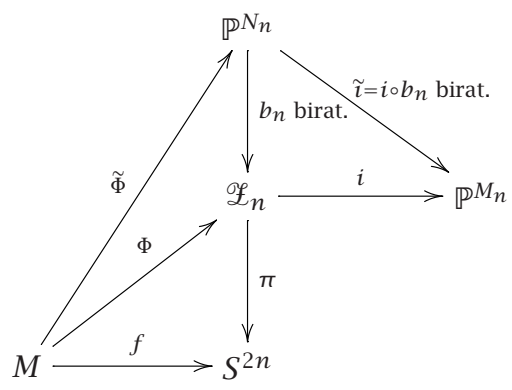

FIGURE 2.1

THEOREM 2.3. There is a one-to-one and onto correspondence

$\{$ twistor lifts $\Phi$ of linearly full superminimal maps $f\}$

$$
\left.\underset{b_{n}^{-1} \circ}{\stackrel{b_{n}^{\circ}}{\longleftrightarrow}} \text { \{solutions } \tilde{\Phi}: M_{g} \longrightarrow \mathbb{P}^{N_{n}} \text { of }(2.17) \text { satisfying }(2.18)\right\} \text {. }
$$

Proof. Recall that $U_{E}$ is the subset of $\mathscr{E}_{n}$ consisting of planes whose orthogonal projection over $E$ is one to one and onto. Let $I_{E}$ be the complement of $U_{E}$. Then $I_{E}$ is a subvariety of $\mathscr{E}_{n}$, of codimension at least one, consisting of those planes having nonempty incidence with $\bar{E}$.

For each choice of a base plane $E$, the map $b_{n}^{-1}$ is regular on $U_{E}$, so for every $\Phi: M_{g} \rightarrow \mathscr{L}_{n}$ intersecting $U_{E}$ we have the claimed correspondence. Thus, we only have to show that, given a plane $F \in \mathscr{E}_{n}$, no superminimal linearly full surface falls completely in $I_{F}$. This is given by the following lemma.

LEMMA 2.4. Let $f: M_{g} \hookrightarrow S^{2 n}$ be a superminimal surface and let $\Phi: M_{g} \rightarrow \mathscr{L}_{n}$ be its twistor lift. If, for all $p \in M_{g}, \Phi(p)$ has nonempty incidence with a fixed plane $F \in \mathscr{L}_{n}$, then $f$ is not linearly full. Consequently, for any choice of base plane $E$, $\Phi$ lies in $U_{E}$ except for finitely many points.

PROOF. We proceed by contradiction. Suppose that $\Phi\left(M_{g}\right) \subset I_{F}$. Let $p$ be a point of $M_{g}$ and choose $E \in \mathscr{Z}_{n}$ so that $\bar{E}$ is not in $I_{E} \cup I_{\Phi(p)}$; then $\bar{E}$ has empty incidence with $F$ and $\Phi(p)$. This guarantees that the curve $\Phi$ lies in $U_{E}$ except for finitely many points, and so does the plane $F$.

Take the parametrization of $\mathscr{L}_{n}$ given in Lemma 2.1 with base plane $E$. Since $F \in U_{E}$, there must be constants $\alpha_{0}, T_{0}$ so that $F$ can be represented as in (2.5). On the other hand, taking a local trivialization $(V, z)$ of $M_{\mathfrak{g}}$, we can represent $\Phi$ by functions $\alpha, T$ satisfying (2.14).

Since $\Phi$ and $F$ have nonempty incidence, there is a locally defined function $v: V \rightarrow \mathbb{C}^{n}$ satisfying

$$
{ }^{t} v \cdot\left(\alpha, I_{n},(-T-A)\right)={ }^{t} v \cdot\left(\alpha_{0}, I_{n},\left(-T_{0}-A_{0}\right)\right) .
$$


Taking derivatives in the last equation and using $-T^{\prime}-A^{\prime}=-\alpha^{\prime} \alpha$, we have

$$
{ }^{t} v \cdot\left(\alpha^{\prime}, O_{n},-\alpha^{\prime}{ }^{t} \alpha\right)+{ }^{t} v^{\prime} \cdot\left(\alpha, I_{n},(-T-A)\right)={ }^{t} v^{\prime} \cdot\left(\alpha_{0}, I_{n},\left(-T_{0}-A_{0}\right)\right) .
$$

This implies ${ }^{t} v \alpha^{\prime}=-{ }^{t} v^{\prime}\left(\alpha-\alpha_{0}\right)$. Substituting in the last component of the last equation, and reordering, we get

$$
{ }^{t} v^{\prime} \cdot\left(\alpha^{t} \alpha-2 \alpha_{0}{ }^{t} \alpha+\alpha_{0}{ }^{t} \alpha_{0}\right)=2^{t} v^{\prime} \cdot\left(T-T_{0}\right)
$$

Multiply the last equation by $v^{\prime}$ from the right. Since $T$ and $T_{0}$ are skewsymmetric, ${ }^{t} v^{\prime} \cdot\left(T-T_{0}\right) \cdot v^{\prime}=0$. Thus, we have

$$
\begin{aligned}
{ }^{t} v^{\prime} \cdot & \left(\alpha^{t} \alpha-2 \alpha_{0}{ }^{t} \alpha+\alpha_{0}{ }^{t} \alpha_{0}\right) v^{\prime} \\
& =\left({ }^{t} v^{\prime} \alpha\right)\left({ }^{t} \alpha v^{\prime}\right)-2\left({ }^{t} v^{\prime} \alpha_{0}\right)\left({ }^{t} \alpha v^{\prime}\right)+\left({ }^{t} v^{\prime} \alpha_{0}\right)\left({ }^{t} \alpha_{0} v^{\prime}\right) \\
& =\left(\left({ }^{t} v^{\prime} \alpha\right)-\left({ }^{t} v^{\prime} \alpha_{0}\right)\right)^{2}=0 .
\end{aligned}
$$

Hence, we must have ${ }^{t} v^{\prime} \alpha={ }^{t} v^{\prime} \alpha_{0}$. Substituting in (2.21), we have

$$
{ }^{t} v \alpha^{\prime}=0
$$

and substituting in (2.20), we have

$$
{ }^{t} v^{\prime} \cdot\left(\alpha, I_{n},(-T-A)\right)={ }^{t} v^{\prime} \cdot\left(\alpha_{0}, I_{n},\left(-T_{0}-A_{0}\right)\right)
$$

That is, $v^{\prime}$ satisfies the same equation as $v$. Proceeding by induction, we will obtain ${ }^{t} v^{(k)} \alpha^{\prime}=0$ for all $k \geq 0$. Differentiating the last equation and substituting terms, we have ${ }^{t} v^{(k)} \alpha^{\prime \prime}=0$ for all $k \geq 0$. Proceeding again by induction, we obtain

$$
{ }^{t} v \alpha^{(\ell)}=0 \quad \forall \ell \geq 1
$$

But this contradicts the condition $W\left(\alpha^{\prime}\right) \not \equiv 0$. Thus, the map $f$ cannot be linearly full.

This completes the proof of Theorem 2.3.

\section{2. $\mathrm{A} \mathrm{Gl}(n)$-action}

DEFINITION 2.5. Let $\widetilde{\Phi}=\left[s: \alpha_{1}: \cdots: \alpha_{n}: \tau_{12}: \cdots: \tau_{n-1, n}\right]$ be a curve in $\mathbb{P}^{N_{n}}$ and let $G$ be an $n \times n$ matrix. We will denote by $G \tilde{\Phi}$ the curve given by

$$
G \tilde{\Phi}:=\left[t: \beta_{1}: \cdots: \beta_{n}: \sigma_{12}: \cdots: \sigma_{1 n}: \cdots: \sigma_{n-1, n}\right]
$$

where $t=s, \beta_{i}=\sum_{j=1}^{n} G_{i j} \alpha_{j}$, and $\sigma_{i j}=\sum_{r, s=1}^{n} G_{i r} G_{j s} \tau_{r s}$. 
In other words, using $\alpha$ and $\beta$ to denote the column vector of the $\alpha_{i}$ and $\beta_{i}$, respectively, and $T$ and $S$ to denote the matrix of the $\tau_{j k}$ and $\sigma_{j k}$, respectively, we have $\beta=G \alpha$ and $S=G T^{t} G$.

Notice that if $\tilde{\Phi}$ satisfies (2.13), then so does $G \tilde{\Phi}$. This set of transformations forms a proper subgroup of the group of linear transformations in $\mathbb{P}^{N_{n}}$ preserving the differential system. The interest of this $\mathrm{Gl}(n)$-action is that it is equivariant through the birational map $b_{n}$ as we will see.

The twistor space $\mathscr{L}_{n}$ can be viewed as the homogeneous space $\mathrm{SO}(2 n+$ $1, \mathbb{C}) / K$, where $K$ is isomorphic to the semidirect product of a nilpotent normal subgroup $L$ and a subgroup $L_{1}$ isomorphic to $\operatorname{Gl}(n, \mathbb{C})$ (see [9] for details). The subgroup $L_{1}$ corresponds to

$$
\left(\begin{array}{ccc}
1 & 0 & 0 \\
0 & H^{-1} & 0 \\
0 & 0 & { }^{t} H
\end{array}\right) \quad \text { with } H \in \operatorname{Gl}(n, \mathbb{C}) .
$$

LEMmA 2.6. The action of $\operatorname{Gl}(n, \mathbb{C})$ in $\mathbb{P}^{N_{n}}$ defined above corresponds, via the birational map $b_{n}$, to the right $\mathrm{Gl}(n, \mathbb{C})$-action on $\mathrm{SO}(2 n+1, \mathbb{C}) / K$ (considered as left cosets).

Proof. We can represent an element of $\mathscr{L}_{n}$ as the equivalence class in $\mathrm{SO}(2 n+1, \mathbb{C}) / K$ of a matrix (see [9])

$$
\left(\begin{array}{ccc}
* & * & * \\
\alpha & I_{n} & -\frac{(\alpha)^{t}(\alpha)}{2}-T \\
* & * & *
\end{array}\right) \in \operatorname{SO}(2 n+1, \mathbb{C})
$$

where the stars denote suitable quantities.

The (right) action on this element by $H \in \operatorname{Gl}(n, \mathbb{C})$ is given by

$$
\left(\begin{array}{ccc}
* & * & * \\
\alpha & H^{-1} & -\frac{(\alpha)^{t}(H \alpha)}{2}-T^{t} H \\
* & * & *
\end{array}\right)
$$

which corresponds to

$$
\left(\begin{array}{ccc}
* & * & * \\
H \alpha & I_{n} & -\frac{(H \alpha)^{t}(H \alpha)}{2}-H T^{t} H \\
* & * & *
\end{array}\right)
$$

modulo multiplication by $H \in \operatorname{Gl}(n, \mathbb{C})$ on the left. Thus the action induced on $\mathscr{L}_{n}$ corresponds to the action on $\mathbb{P}^{N_{n}}$ defined above. 
REMARK 2.7. This $\operatorname{Gl}(n, \mathbb{C})$-action is the restriction to $\mathscr{L}_{n}$ of the linear action of $L_{1} \subset \mathrm{Gl}(2 n+1, \mathbb{C})$ on $\mathbb{P}\left(\Lambda^{n}\left(\mathbb{C}^{2 n+1}\right)\right) \simeq \mathbb{P}^{M_{n}}$ via the exterior representation of $\mathrm{Gl}(2 n+1, \mathbb{C})$. Therefore, the degree of any curve in any of these spaces is preserved by the action.

REMARK 2.8. Notice that the planes $E$ and $\bar{E}$ are invariant under this $\operatorname{Gl}(n$, $\mathbb{C}$ )-action. In particular, the dimension of the intersection of $\bar{E}$ with any other plane is also invariant under the action.

3. The moduli spaces. Recall that, given a branched superminimal immersion $f: M_{g} \rightarrow S^{2 n}$, the degree of $f$ can be defined as the degree of its twistor lift into $\mathscr{L}$ or as Area $f\left(M_{g}\right) / 4 \pi$ (see [1]). The degree is the fundamental invariant of these maps. We will use the following notation:

(i) $\operatorname{Supm}_{d}^{m}\left(M_{g}, S^{2 n}\right)$ : moduli space of superminimal surfaces in $S^{2 n}$ of degree $d$ and genus $g$, lying in a $(2 m+1)$-subspace of $\mathbb{R}^{2 n+1}$;

(ii) $\operatorname{Supm}_{d}^{f}\left(M_{g}, S^{2 n}\right)$ : subset of $\operatorname{Supm}_{d}^{n}\left(M_{g}, S^{2 n}\right)$ consisting of linearly full surfaces.

Notice that, by definition,

$$
\operatorname{Supm}_{d}^{n}\left(M_{g}, S^{2 n}\right) \supseteq \operatorname{Supm}_{d}^{n-1}\left(M_{g}, S^{2 n}\right) \supseteq \cdots \supseteq \operatorname{Supm}_{d}^{1}\left(M_{g}, S^{2 n}\right) .
$$

The corresponding objects for $\mathscr{E}_{n}$ are

(i) $\mathrm{HH}_{d}^{m}\left(M_{g}, \mathscr{L}_{n}\right)$ : moduli space of holomorphic and horizontal maps $\Phi$ : $M_{g} \rightarrow \mathscr{L}_{n}$ of degree $d$ consisting of planes in $\mathscr{L}_{n}$ lying in a $2 m+1$ subspace of $\mathbb{C}^{2 n+1}$;

(ii) $\mathrm{HH}_{d}^{f}\left(M_{g}, \mathscr{E}_{n}\right)$ : subset of $\mathrm{HH}_{d}^{n}\left(M_{g}, \mathscr{E}_{n}\right)$ corresponding to linearly full maps.

Using the Plücker embedding of $\mathscr{E}_{n}$ into $\mathbb{P}^{M_{n}}$, whose degree is 2 (see [1, page 92] and [12, page 129]), we can think of $\mathrm{HH}_{d}^{m}\left(M_{g}, \mathscr{L}_{n}\right)$ and $\mathrm{HH}_{d}^{f}\left(M_{g}, \mathscr{L}_{n}\right)$ as subvarieties of $H_{0}(M, \mathscr{L}(2 d))^{M_{n}}$ consisting of sections without common zeroes that satisfy the conditions of lying in $\mathscr{L}_{n}$, of being horizontal, and of being linearly full in the case of $\mathrm{HH}_{d}^{f}\left(M_{g}, \mathscr{E}_{n}\right)$. These are all algebraic conditions. Therefore, $\mathrm{HH}_{d}^{m}\left(M_{g}, \mathscr{E}_{n}\right)$ and $\mathrm{HH}_{d}^{f}\left(M_{g}, \mathscr{E}_{n}\right)$ are algebraic varieties, although we have a priori no information about their structure.

The relationship between these objects is as follows (see [7] for details). Given $f \in \operatorname{Supm}_{d}^{f}\left(M_{g}, S^{2 n}\right)$, we can uniquely construct its twistor lift. We have a map

$$
\operatorname{Supm}_{d}^{f}\left(M_{g}, S^{2 n}\right) \underset{f \longrightarrow \Phi}{\stackrel{\text { twistor lift }}{\longrightarrow}} \mathrm{HH}_{d}^{f}\left(M_{g}, \mathscr{L}_{n}\right)
$$

which satisfies $\pi \circ \Phi= \pm f$, where $\pi$ is the projection defined in (2.7). It is important to notice that both $f$ and $-f$ have the same twistor lift, so that 
the map

$$
\begin{gathered}
\mathrm{HH}_{d}^{n}\left(M_{g}, \mathscr{L}_{n}\right) \stackrel{\Pi^{+}}{\longrightarrow} \operatorname{Supm}_{d}^{n}\left(M_{g}, S^{2 n}\right) \\
\Phi \longrightarrow f=\pi \circ \Phi
\end{gathered}
$$

is not surjective. We can then consider the maps

$$
\begin{aligned}
\operatorname{HH}_{d}^{n}\left(M_{g}, \mathscr{L}_{n}\right) & \stackrel{\Pi^{+}}{\longrightarrow} \operatorname{Supm}_{d}^{n}\left(M_{g}, S^{2 n}\right) \\
\Phi & \longrightarrow \pi \circ \Phi, \\
\mathrm{HH}_{d}^{n}\left(M_{g}, \mathscr{E}_{n}\right) & \stackrel{\Pi^{-}}{\longrightarrow} \operatorname{Supm}_{d}^{n}\left(M_{g}, S^{2 n}\right) \\
\Phi & \longrightarrow-\pi \circ \Phi .
\end{aligned}
$$

We denote by $\operatorname{Supm}_{d}^{+}\left(M_{g}, S^{2 n}\right)$ and $\operatorname{Supm}_{d}^{-}\left(M_{g}, S^{2 n}\right)$ the images of $\Pi^{+}$and $\Pi^{-}$in $\operatorname{Supm}_{d}^{n}\left(M_{g}, S^{2 n}\right)$, respectively. We have (see [7])

$$
\begin{aligned}
\operatorname{Supm}_{d}^{f}\left(M_{g}, S^{2 n}\right) & =\Pi^{+}\left(\mathrm{HH}_{d}^{f}\left(M_{g}, S^{2 n}\right)\right) \cup \Pi^{-}\left(\mathrm{HH}_{d}^{f}\left(M_{g}, S^{2 n}\right)\right), \\
\operatorname{Supm}_{d}^{n}\left(M_{g}, S^{2 n}\right) & =\operatorname{Supm}_{d}^{+}\left(M_{g}, S^{2 n}\right) \cup \operatorname{Supm}_{d}^{-}\left(M_{g}, S^{2 n}\right), \\
\operatorname{Supm}_{d}^{n-1}\left(M_{g}, S^{2 n}\right) & =\operatorname{Supm}_{d}^{+}\left(M_{g}, S^{2 n}\right) \cap \operatorname{Supm}_{d}^{-}\left(M_{g}, S^{2 n}\right) .
\end{aligned}
$$

Now we define the corresponding objects in $\mathbb{P}^{N_{n}}$.

(i) $\mathrm{PD}_{d}^{m}\left(M_{g}, \mathbb{P}^{N_{n}}\right)$ : moduli space of holomorphic maps $\tilde{\Phi}: M_{g} \rightarrow \mathbb{P}^{N_{n}}$ of degree $d$ satisfying the differential system (2.13) and lying in an $N_{m^{-}}$ subspace of $\mathbb{P}^{N_{n}}$.

(ii) $\mathrm{PD}_{d}^{f}\left(M_{g}, \mathbb{P}^{N_{n}}\right)$ : subset of $\mathrm{PD}_{d}^{n}\left(M_{g}, \mathbb{P}^{N_{n}}\right)$ of maps satisfying (2.18).

The birational map $b_{n}: \mathbb{P}^{N_{n}} \rightarrow \mathscr{L}_{n}$ (which depends on the base plane chosen) induces maps

$$
\begin{aligned}
\mathscr{B}_{n}^{E}: \mathrm{PD}_{d}^{f}\left(M_{g}, \mathbb{P}^{N_{n}}\right) & \longrightarrow \bigcup_{k=0}^{\infty} \mathrm{HH}_{k}^{f}\left(M_{g}, \mathscr{L}_{n}\right) \\
\widetilde{\Phi} & \longrightarrow \Phi=b_{n} \circ \widetilde{\Phi} \\
\mathscr{C}_{n}^{E}: \mathrm{HH}_{d}^{f}\left(M_{g}, \mathscr{L}_{n}\right) & \longrightarrow \bigcup_{k=0}^{\infty} \operatorname{PD}_{k}^{f}\left(M_{g}, \mathbb{P}^{N_{n}}\right) \\
\Phi & \longrightarrow \widetilde{\Phi}=b_{n}^{-1} \circ \Phi .
\end{aligned}
$$

THEOREM 3.1. The moduli spaces $\mathrm{PD}_{d}^{f}\left(M_{g}, \mathbb{P}^{N_{n}}\right)$ and $\mathrm{HH}_{d}^{f}\left(M_{g}, \mathscr{E}_{n}\right)$ are birationally equivalent via the maps $\mathscr{C}_{E}$, with inverses $\mathscr{B}_{E}$.

In addition, the image of the map $\mathscr{B}_{n}^{E}$ lies in

$$
\bigcup_{k=d}^{2 d n} \operatorname{HH}_{k}^{f}\left(M_{g}, \mathscr{Z}_{n}\right)
$$


and the image of the map $\mathscr{C}_{n}^{E}$ lies in

$$
\bigcup_{k=0}^{d} \operatorname{PD}_{k}^{f}\left(M_{g}, \mathbb{P}^{N_{n}}\right) .
$$

REMARK 3.2. We do not know a priori if any of these moduli spaces is irreducible or even if it has pure dimension. Hence, our claim is that, for each $\Phi \in \mathrm{HH}_{d}^{f}\left(M_{g}, \mathscr{E}_{n}\right)$, the map $\mathscr{C}_{E}$ is a birational map from the irreducible component where $\Phi$ lies in the irreducible component of $\operatorname{PD}_{d}^{f}\left(M_{g}, \mathbb{P}^{N_{n}}\right)$, where $\widetilde{\Phi}=\mathscr{C}_{E}(\Phi)$ lies, for some base plane $E \in \mathscr{L}_{n}$.

If the spaces have pure dimension, then, for any $E \in \mathscr{L}_{n}, \mathscr{C}_{E}$ is a birational correspondence between the irreducible components of the moduli spaces, with inverse $\mathscr{B}_{E}$.

Proof. We need several technical results. We give here a summary of what we are going to do.

(A) Find conditions on $\tilde{\Phi}$ that guarantee that $\operatorname{deg}(\tilde{\Phi})=\operatorname{deg}\left(b_{n} \circ \tilde{\Phi}\right)$.

(B) Using (A), find conditions on $\Phi$ that guarantee that $\operatorname{deg}(\Phi)=\operatorname{deg}\left(b_{n}^{-1} \circ \Phi\right)$.

(C) Show that, for suitable choices of $E$, these conditions hold in Zariski open subsets of each irreducible component of $\mathrm{HH}_{d}^{f}\left(M_{\mathfrak{g}}, \mathscr{Z}_{n}\right)$.

This will imply that, for some $E$, the map $\mathscr{C}_{n}^{E}$ is regular in Zariski open subsets of the irreducible components of $\mathrm{HH}_{d}^{f}\left(M_{g}, \mathscr{Z}_{n}\right)$. Since $\mathscr{C}_{n}^{E}$ is injective, it gives the claimed correspondence between the irreducible components.

(A) Conditions on $\tilde{\Phi}$. Consider the situation of Figure 2.1. After completing the curve $\tilde{\imath} \circ \tilde{\Phi}$, defined at first only at the points where $\tilde{\imath}$ is a morphism, we must have $\tilde{\imath} \circ \tilde{\Phi}=i \circ \Phi$. Since the degree of the Plücker embedding $\mathscr{L}_{n} \rightarrow \mathbb{P}^{M_{n}}$ is 2 , we want to find under what conditions the degree of $\tilde{\imath} \circ \tilde{\Phi}$ is equal to twice the degree of $\tilde{\Phi}$.

The components in $\mathbb{P}^{M_{n}}$ of the map $\tilde{\imath}: \mathbb{P}^{N_{n}} \rightarrow \mathbb{P}^{M_{n}}$ are defined explicitly as the determinants of the $n \times n$ minors of the matrix

$$
\left(\frac{\alpha}{s}, I_{n},\left(-\frac{T}{s}-\frac{\alpha^{t} \alpha}{2 s^{2}}\right)\right)
$$

Let $H^{\prime}$ be the hyperplane in $\mathbb{P}^{M_{n}}$ given by the vanishing of the component corresponding to the determinant of the minor $I_{n}$ in (3.9) and let $H$ be the hyperplane in $\mathbb{P}^{N_{n}}$ given by the vanishing of the first component (the one corresponding to $s$ ).

Let $\sum_{i=1}^{k} m_{i} p_{i}$ be the pullback under the map $\widetilde{\Phi}$ of the hyperplane divisor corresponding to $H$ and let $\sum_{i=1}^{k} m_{i}^{\prime} p_{i}$ be the pullback under the map $\tilde{\imath} \circ \tilde{\Phi}$ of the hyperplane divisor corresponding to $H^{\prime}$. Note that the points where the divisor is supported are the same in both cases since, after taking local charts and homogenizing in $\mathbb{P}^{M_{n}}$, the component corresponding to the determinant of the minor $I_{n}$ will have the form $s^{\ell}$, for some $\ell$. 
We have $\operatorname{deg}(\tilde{\Phi})=\sum_{j=1}^{k} m_{j}$ and $\operatorname{deg}(\tilde{\imath} \circ \tilde{\Phi})=\sum_{j=1}^{k} m_{j}^{\prime}$.

Proposition 3.3. (a) Let $\sum_{i=1}^{k} m_{i} p_{i}$ be the pullback under the map $\widetilde{\Phi}$ of the hyperplane divisor corresponding to $H$ (i.e., $s=0$ ). If $\alpha\left(p_{i}\right) \neq 0$ for all $i$, then

$$
\operatorname{deg}(\widetilde{\Phi})=\frac{\operatorname{deg}(\tilde{\imath} \circ \widetilde{\Phi})}{2}=\operatorname{deg}(\Phi)
$$

In other words, if $\alpha \neq 0$ when $s=0$, then the degrees of $\Phi$ and $\tilde{\Phi}$ are equal.

(b) For arbitrary $\Phi, \operatorname{deg}(\tilde{\Phi}) \leq \operatorname{deg}(\tilde{\imath} \circ \tilde{\Phi}) / 2=\operatorname{deg}(\Phi) \leq 2 n \operatorname{deg}(\tilde{\Phi})$.

Proof. We have to show that, for any $p \in\left\{p_{1}, p_{2}, \ldots, p_{k}\right\}$, the map $\tilde{\imath} \circ \tilde{\Phi}$ has as a denominator at most $s^{2}$ in each of its components. So the question becomes local, and we can do the proof about a general $p$.

Let $p \in\left\{p_{1}, \ldots, p_{k}\right\}$ and let $m$ be its corresponding multiplicity. Let $(U, z)$ be a local trivialization about $p \in M_{g}$ so that we can write

$$
\begin{gathered}
s=z^{m}, \\
\alpha=\sum_{k=0}^{\infty} a_{k} z^{k} \quad\left(a_{k} \in \mathbb{C}^{n}\right), \\
T=\sum_{k=0}^{\infty} t_{k} z^{k} \quad\left(t_{k} \in \mathcal{M}_{n \times n}\right),
\end{gathered}
$$

where we assume that $s, \alpha$, and $T$ have no common zeroes.

The equation $d \alpha^{t} \alpha-\alpha d^{t} \alpha=2(s d T-T d s)$ can be written as

$$
\sum_{n=0}^{\infty} \sum_{r=0}^{n}(n+1-r)\left(a_{n+1-r}{ }^{t} a_{r}-a_{r}{ }^{t} a_{n+1-r}\right) z^{n}=2 \sum_{k=0}^{\infty}(k-m) t_{k} z^{k+m-1} .
$$

This implies

$$
\begin{gathered}
\sum_{r=0}^{n}(n+1-r)\left(a_{n+1-r}{ }^{t} a_{r}-a_{r}{ }^{t} a_{n+1-r}\right)=0 \quad \text { if } n<m-1, \\
\sum_{r=0}^{n}(n+1-r)\left(a_{n+1-r}{ }^{t} a_{r}-a_{r}{ }^{t} a_{n+1-r}\right)=2(n+1-2 m) t_{n-(m-1)} \quad \text { if } n \geq m-1 .
\end{gathered}
$$

Equation (3.13) implies that there exist numbers $\lambda_{i}$ such that

$$
a_{i}=\lambda_{i} a_{0} \text { for } 0 \leq i \leq m-1
$$

Now we look at (3.14). Observe that, for $m-1 \leq n \leq 2 m-2$, either $r \leq m-1$ or $n+1-r \leq m-1$. Thus, using (3.15) and changing summation variables, 
we obtain

$$
\begin{aligned}
a_{0}{ }^{t}( & \left.\sum_{r=m}^{n}(n+1-r) \lambda_{n+1-r} a_{0} a_{r}-\sum_{r=0}^{m-1}(n+1-r) \lambda_{r} a_{0} a_{n+1-r}\right) \\
& -\left(\sum_{r=m}^{n}(n+1-r) \lambda_{n+1-r} a_{0} a_{r}-\sum_{r=0}^{m-1}(n+1-r) \lambda_{r} a_{0} a_{n+1-r}\right){ }^{t} a_{0} \\
= & 2(n+1-2 m) t_{n+1-m} \text { for } m-1 \leq n \leq 2 m-2 .
\end{aligned}
$$

Define

$\mathscr{A}=\sum_{n=m-1}^{2 m-2}\left(\sum_{r=m}^{n} \frac{n+1-r}{n+1-2 m} \lambda_{n+1-r} a_{0} a_{r}-\sum_{r=0}^{m-1} \frac{n+1-r}{n+1-2 m} \lambda_{r} a_{0} a_{n+1-r}\right) z^{n+1-m}$.

Then $T$ can be written as

$$
T=\frac{1}{2}\left(a_{0}^{t} \mathscr{A}-\mathscr{A}^{t} a_{0}\right)+s T_{1}
$$

where $T_{1}$ is a holomorphic matrix-valued function. Now, using (3.15), $\alpha$ can be written as

$$
\alpha=\Lambda \cdot a_{0}+s A_{1}
$$

where $\Lambda=\sum_{i=0}^{m-1} \lambda_{i} z^{i}$ and $A_{1}=\sum_{i=m}^{\infty} a_{i} z^{i}$ are holomorphic $\mathbb{C}$ - and $\mathbb{C}^{n}$-valued functions, respectively.

Then the matrix $\left(\alpha / s, I_{n},-T / s-\alpha^{t} \alpha / 2 s^{2}\right)$ can be written as

$$
\left(\frac{\Lambda \cdot a_{0}}{s}+A_{1}, I_{n},-\frac{\Lambda^{2} \cdot a_{0}{ }^{t} a_{0}}{2 s^{2}}-\frac{a_{0}{ }^{t}\left(\Lambda \cdot A_{1}-\mathscr{A}\right)+\left(\Lambda \cdot A_{1}+\mathscr{A}\right)^{t} a_{0}}{s}-A_{1}{ }^{t} A_{1}+T_{1}\right) .
$$

Defining $\xi=\Lambda \cdot a_{0}, \beta=\Lambda \cdot A_{1}-\mathscr{A}$, and $\gamma=\Lambda \cdot A_{1}+\mathscr{A}$, which are all holomorphic, and denoting holomorphic terms by "Hol," the last matrix adopts the form

$$
\left(\frac{\xi}{s}+\mathrm{Hol}, I_{n},-\frac{\xi^{t} \xi}{2 s^{2}}-\frac{\xi^{t} \beta+\gamma^{t} \xi}{2 s}+\mathrm{Hol}\right)
$$

The following lemma completes the proof of statement (a).

LEMMA 3.4. The highest power of s that appears in the denominator of any of the $n \times n$ minors of a matrix of the form

$$
\left(\frac{\xi}{s}+\mathrm{Hol}, I_{n},-\frac{\xi^{t} \xi}{2 s^{2}}-\frac{\xi^{t} \beta+\gamma^{t} \xi}{2 s}+\mathrm{Hol}\right)
$$

is 2 . 
Proof. The proof is elementary but tedious. Notice the following relations. If $\xi_{i} \neq 0$,

$$
\begin{aligned}
\left(\frac{s \beta_{j}}{\xi_{i}}-\frac{s \beta_{i}}{\xi_{i}^{2}} \xi_{j}+\frac{\xi_{j}}{\xi_{i}}\right)\left(-\frac{\xi \xi_{i}}{2 s^{2}}-\frac{\xi \beta_{i}+\gamma \xi_{i}}{2 s}+\text { Hol }\right) & =-\frac{\xi \xi_{j}}{2 s^{2}}-\frac{\xi \beta_{j}+\gamma \xi_{j}}{2 s}+\text { Hol } \\
& \frac{2 s}{\xi_{i}}\left(-\frac{\xi \xi_{i}}{2 s^{2}}-\frac{\xi \beta_{i}+\gamma \xi_{i}}{2 s}+\text { Hol }\right)=-\frac{\xi}{s}+\text { Hol }
\end{aligned}
$$

where Hol denotes "holomorphic terms."

If $\beta_{i} \neq 0$,

$$
\frac{\beta_{i}}{\beta_{j}}\left(\frac{\xi \beta_{i}}{2 s}+\text { Hol }\right)=\frac{\xi \beta_{j}}{2 s}+\text { Hol }
$$

Using these formulas, one can do column operations in each of the minors to reduce it either to a matrix with $s^{2}$ in the denominator of only one column or to a matrix with $s$ in the denominator of only two columns. We omit the details.

To prove statement (b) of the proposition, notice that, for arbitrary $\widetilde{\Phi}$, some of the minors of the matrix (3.9) have the form $\alpha_{i}^{2} / s^{2}$ and others have the form $\tau_{j k}^{2} / s^{2}$, so at least one of the minors will have an $s^{2}$ in the denominator. On the other hand, the denominator of the highest possible degree can be roughly estimated as $s^{2 n}$. This proves statement (b).

(B) Conditions on $\Phi$. Recall that $I_{E}$ is the set of all planes in $\mathscr{E}_{n}$ having nontrivial incidence with $\bar{E}$.

Proposition 3.5. Let $\Phi: M_{g} \rightarrow \mathscr{L}_{n}$ be the twistor lift of a superminimal curve $f: M_{g} \hookrightarrow S^{2 n}$. Let $E$ be a base plane and let $\left\{p_{1}, \ldots, p_{k}\right\} \in M_{g}$ be the set of points in $M_{g}$ such that $\Phi\left(p_{i}\right) \in I_{E}$. If the dimension of the incidence between $\bar{E}$ and $\Phi\left(p_{i}\right)$ (considered as $n$-planes in $\mathbb{C}^{2 n+1}$ ) is 1 for every point $p_{i}$, then the hypotheses of Proposition 3.3 are satisfied. In particular, the degrees of $\Phi$ and $\widetilde{\Phi}$ are equal.

Proof. Take a point $p \in\left\{p_{1}, \ldots, p_{k}\right\}$. Then $\Phi(p)$ has the form $\bar{F}_{1} \wedge F_{2} \wedge$ $\cdots \wedge F_{n}$, where $\bar{F}_{1} \in \bar{E}$ and $F_{j} \notin \bar{E}, 2 \leq j \leq n$. Using the $\operatorname{Gl}(n, \mathbb{C})$ action we can assume, without loss of generality, that $\Phi(p)=\bar{E}_{1} \wedge F_{2} \wedge \cdots \wedge F_{n}$ (note that by Lemma 2.6 and Remarks 2.7 and 2.8, this action preserves both the degree of the curve and the dimension of the incidence of the curve with $E$ and $\bar{E}$ ). The vectors $F_{j} \in E, 2 \leq j \leq n$, can be written in the form $F_{j}=E_{j}+f_{0} E_{0}+\sum_{k=1}^{n} f_{k} \bar{E}_{k}$, for some coefficients $f_{k}, 0 \leq k \leq n$, in view of (2.2).

This implies that the component of $\tilde{\imath} \circ \tilde{\Phi}$, in homogeneous coordinates of $\mathbb{P}^{M_{n}}$, corresponding to $\bar{E}_{1} \wedge E_{2} \wedge \cdots \wedge E_{n}$, is 1 after homogenizing in $\mathbb{P}^{M_{n}}$. Now, 
when we represent the curve in terms of $s, \alpha_{i}, \tau_{j k}$ about $p$, this component corresponds to the minor formed by columns 3 through $n+2$ of the matrix

$$
\left(\frac{\alpha}{s}, I_{n},\left(-\frac{T}{s}-\frac{\alpha^{t} \alpha}{2 s^{2}}\right)\right)
$$

which is $-\alpha_{1}^{2} / 2 s^{2}$. Hence, denoting the homogenizing factor in $\mathbb{P}^{M_{n}}$ by $h$, we must have $-h \cdot \alpha_{1}^{2} / 2 s^{2} \neq 0$ at $p$.

However, the homogenizing factor $h$ has to have at least the degree of $s^{2}$. This implies that $\alpha(p) \neq 0$. Since $p$ was arbitrary, we must have that the curve $\widetilde{\Phi}$ satisfies the hypotheses of Proposition 3.3.

(C) THE SET OF $\Phi$ THAT SATISFIES THE CONDITIONS OF PROPOSITION 3.5 IS A ZARISKI OPEN SUBSET OF EACH IRREDUCIBLE COMPONENT OF $\mathrm{HH}_{d}^{f}\left(M_{g}, \mathscr{L}_{n}\right)$. We can restate Proposition 3.5 as follows. The maps $\Phi$ in each irreducible component $\mathscr{I}$ of $\mathrm{HH}_{d}^{f}\left(M_{g}, \mathscr{L}_{n}\right)$ that at some point have incidence of dimension greater than one with a fixed plane $\bar{E} \in \mathscr{L}_{n}$ form a proper subvariety of the variety of maps from $M_{g}$ to $\mathscr{E}_{n}$. Thus, given a base plane $E$, either no maps in this irreducible component $\mathscr{I}$ of $\mathrm{HH}_{d}^{f}\left(M_{g}, \mathscr{L}_{n}\right)$ satisfy the hypotheses of Proposition 3.5 or the set of maps that satisfy the hypotheses of Proposition 3.5 form a Zariski open subset of $\mathscr{I}$.

Hence, to complete the proof of Theorem 3.1, we only need to prove that, given $\Phi \in \mathrm{HH}_{d}^{f}\left(M_{g}, \mathscr{L}_{n}\right)$, we can find a base plane $E$ so that $\Phi$ and $E$ satisfy the hypotheses of Proposition 3.5. Then the map $\mathscr{C}_{E}$ will be regular and injective in a Zariski open subset of the irreducible component where $\Phi$ belongs, and this gives the desired birational correspondence between the irreducible components.

Proposition 3.6. Let $\Phi: M_{g} \rightarrow \mathscr{E}_{n}$ be the twistor lift of a linearly full superminimal surface $f: M_{g} \hookrightarrow S^{2 n}$. Then there is a plane $F$ in $\mathbb{C}^{2 n+1}$ so that the incidence between $\Phi$ and $F$ has dimension less than two at all points.

The proof will be immediate after the following lemmas.

LEMMA 3.7. Let $F$ be an isotropic $n$-plane in $\mathbb{C}^{2 n+1}$, that is, an element of $\mathscr{L}_{n}$. Let $J_{F}$ be the subset of $\mathscr{L}_{n}$ consisting of those planes whose incidence with $F$ is at least 2-dimensional. Then $J_{F}$ is a subvariety of $\mathscr{L}_{n}$ of codimension at least 2.

Proof. Take a unitary basis in $\mathbb{C}^{2 n+1}$ so that $F=E_{1} \wedge \cdots \wedge E_{n}$ and take the parametrization given in Lemma 2.1 using this base plane. Then the intersection of $J_{F}$ with the Zariski open subset $U_{F} \subseteq \mathscr{L}_{n}$ is given, in terms of the parametrization, by

$$
\{(\alpha, T): \operatorname{rank}(\alpha \mid T) \leq n-2\} .
$$


The condition rank $(\alpha \mid T) \leq n-2$ is clearly algebraic and it involves at least two independent algebraic conditions on the $\alpha_{i}, \tau_{j k}$ (namely that all the $(n-$ $1) \times(n-1)$ minors are 0$)$. Therefore, $J_{F}$ is a subvariety of $\mathscr{E}_{n}$ of codimension at least 2 .

LEMMA 3.8. Consider the set

$$
\mathscr{W}=\bigcup_{p \in M_{g}} J_{\Phi(p)} .
$$

Then $W$ is a subvariety of $\mathscr{L}_{n}$ of codimension at least 1.

Proof. Consider the set $\mathscr{V} \subseteq M_{g} \times \mathscr{L}_{n}$ given by

$\mathscr{V}=\{(p, P)$ : the incidence between $\Phi(p)$ and $P$ has dimension at least 2$\}$

and let $\pi_{i}$ denote projection over the $i$ th factor. Since the incidence condition defining $\mathscr{V}$ is algebraic (essentially it can be seen as the vanishing of the determinant of some matrices), $\mathscr{V}$ must be a projective variety. In view of the previous lemma, the dimension of the fiber of the projection $\pi_{1}$ over $M_{g}$ is at most $\operatorname{dim}\left(\mathscr{E}_{n}\right)-2$. Therefore, the dimension of $\mathscr{V}$ is at most $\operatorname{dim}\left(\mathscr{E}_{n}\right)-1$ (also note that, locally, $\mathscr{V}$ is basically a product with some identifications accounting for the planes whose incidence with the curve has dimension greater than 1 at two different points of the curve).

Now, $\mathscr{W}$ is the projection of $\mathscr{V}$ over $\mathscr{E}_{n}$, so $\mathscr{W}$ is a projective subvariety of $\mathscr{E}_{n}$. To compute its dimension, note that the fiber of the projection $\pi_{2}$ over $\mathscr{E}_{n}$ is a discrete set since each element of $\mathscr{E}_{n}$, viewed as a plane, has nonempty incidence with the curve at only finitely many points (see the proof of Lemma 2.4). Therefore the dimension of $\mathscr{W}$ is at most $\operatorname{dim}\left(\mathscr{E}_{n}\right)-1$. In other words, its codimension is at least 1 , as desired.

Proof of Proposition 3.6. The previous lemma and the fact that $U_{E}$ is a Zariski open subset in $\mathbb{P}^{N_{n}}$ show that the set of planes whose incidence with $\Phi$ has dimension 1 at all points of intersection is actually a dense subset of $\mathscr{L}_{n}$.

END OF THE PROOF OF THEOREM 3.1. Take $\Phi \in \mathrm{HH}_{d}^{f}\left(M_{g}, \mathscr{Z}_{n}\right)$. Use Proposition 3.6 to find $F$ having incidence of dimension at most 1 with $\Phi$. Then $E=\bar{F}$ and $\Phi$ satisfy the hypotheses of Proposition 3.5. This implies that these hypotheses are also satisfied in a Zariski open set containing $\Phi$. Therefore the map $\mathscr{C}_{E}(\Phi)$ is regular in a Zariski open subset of the irreducible component where $\Phi$ lies. Finally, $\mathscr{C}$ is injective, so it is a birational map with inverse $\mathscr{B}_{E}$.

Note that if we fix the base plane $E$ beforehand, Lemmas 3.7 and 3.8 guarantee that there will always be a $\Phi \in \operatorname{HH}_{d}^{f}\left(M_{g}, \mathscr{L}_{n}\right)$ lying in the regular part of $\mathscr{C}_{E}$. Since this regular part is open, $\mathscr{C}_{E}$ will be birational between the components of top dimension. 
4. Existence of superminimal surfaces of arbitrary large area. All the results of the previous sections would be quite useless unless we knew that the aforementioned moduli spaces are not empty. In this section, we show that there are superminimal surfaces of arbitrary high degree. There are previous existence proofs; in [1], there is an explicit construction of full minimal maps from $S^{2} \rightarrow S^{2 n}$ with arbitrarily large area, but this method does not work for general genus; in [5, 9], they consider general genus, but they only mention the ramification degree of the curve and not the area. The methods we use are similar to the ones used in $[3,4,5,9]$, but we have the additional fact that we can control the degree.

THEOREM 4.1. Let $M_{g}$ be a Riemann surface of genus $g$. Then, for any $n$, there exist linearly full superminimal maps $f: M_{g} \rightarrow S^{2 n}$ with arbitrarily large area.

Proof. In view of Proposition 3.3, we have Area $f\left(M_{g}\right) / 4 \pi=\operatorname{deg}(\Phi) \geq$ $\operatorname{deg}(\tilde{\Phi})$, so we need to show that there exist maps $M_{g} \rightarrow \mathbb{P}^{N_{n}}$ with arbitrarily large degree that are integrals of the differential system (2.13) and that satisfy the conditions for linear fullness.

After homogenizing in $\mathbb{P}^{N_{n}}$, the problem reduces to finding a meromorphic map

$$
\begin{gathered}
\tilde{\Phi}: M_{\mathcal{g}} \rightarrow \mathbb{P}^{N_{n}} \\
p \rightarrow\left[1: \alpha_{1}(p): \cdots: \alpha_{n}(p): \tau_{12}(p): \cdots: \tau_{n-1, n}(p)\right]
\end{gathered}
$$

of arbitrarily large degree, and satisfying

$$
\alpha_{j} d \alpha_{i}-\alpha_{i} d \alpha_{j}=2 d \tau_{i j}
$$

and the additional condition that guarantees linear fullness.

For convenience, we define $\sigma_{i j}=\alpha_{i} \alpha_{j}+2 \tau_{i j}, i<j$. Then we have

$$
d \sigma_{i j}=\alpha_{j} d \alpha_{i}+\alpha_{i} d \alpha_{j}+2 d \tau_{i j}=2 \alpha_{j} d \alpha_{i}
$$

In other words, if we find meromorphic functions $\alpha_{i}, \sigma_{j k}$ on $M_{\mathfrak{g}}$ satisfying

$$
d \sigma_{i j}=2 \alpha_{j} d \alpha_{i}, \quad i<j
$$

then, defining $\tau_{i j}=\left(\sigma_{i j}-\alpha_{i} \alpha_{j}\right) / 2$, we will obtain a map $\tilde{\Phi}$ as specified above.

The proof is a variation of the argument in [4]. The idea is to make use of the Riemann-Roch theorem to ensure that we can choose meromorphic functions on $M_{g}$ from a large enough vector space of functions.

Let $p_{1}, p_{2}, \ldots, p_{n}$ be $n$ distinct points on $M_{g}$. Choose $\alpha_{1}$ meromorphic on $M_{g}$ such that

$$
\left(\alpha_{1}\right)=-N_{1} p_{1}+D
$$


where $(g)$ denotes the divisor of a meromorphic function $g, N_{1}$ is a positive integer, and $D=\sum_{i=1}^{N_{1}} q_{i}$ is an effective divisor with degree greater than or equal to 2 (so $N_{1} \geq 2$ ).

Consider the divisor $N_{2} p_{2}-D$, where $N_{2}$ is a positive integer. By RiemannRoch,

$$
\begin{aligned}
\operatorname{dim}\left(\mathscr{L}\left(N_{2} p_{2}-D\right)\right) & =i\left(N_{2} p_{2}-D\right)+\operatorname{deg}\left(N_{2} p_{2}-D\right)+1-g \\
& \geq N_{2}-C(D, g),
\end{aligned}
$$

where $C(D, g)$ is a constant depending only on the divisor $D$ and the genus $g$.

Let $\left\{\gamma_{1}, \gamma_{2}, \ldots, \gamma_{2 g}\right\}$ be a basis for the first homology of $M_{g}$. A necessary and sufficient condition for a form $\omega$ to be exact is that it has no residues and that

$$
\int_{\gamma_{i}} \omega=0 \quad \forall i
$$

Consider the linear map $Q_{2}: \mathscr{L}\left(N_{2} p_{2}-D\right) \rightarrow \mathbb{C}^{2 g}$ defined by

$$
f \rightarrow\left(\int_{\gamma_{1}} f d \alpha_{1}, \ldots, \int_{\gamma_{2 g}} f d \alpha_{1}\right)
$$

Since the dimension of $\mathscr{L}\left(N_{2} p_{2}-D\right)$ is greater than or equal to $N_{2}-C(D, g)$, by taking $N_{2}$ large enough we can guarantee that $Q_{2}$ has a large kernel and that $f d \alpha_{1}$ does not have residues at $p_{1}$. Also, taking $N_{2}$ even larger if needed, we can also guarantee that the quantities $f\left(r_{1}\right), f\left(r_{2}\right), \ldots, f\left(r_{N_{1}}\right)$, where $\left\{r_{1}, r_{2}, \ldots\right.$, $\left.r_{N_{1}}\right\}=\alpha_{1}^{-1}(a)$ with $a$ some point in the image of $\alpha_{1}$, are all distinct for some element $f$ in the kernel of $Q_{2}$ (this condition will not be used until the end of the proof).

Choose $\alpha_{2}=f$. Since $\alpha_{2}$ is in the kernel of $Q_{2}$, the differential $\alpha_{2} d \alpha_{1}$ must be exact. Since $\alpha_{2} d \alpha_{1}$ has only two poles ( $p_{1}$ and $p_{2}$ ) and it has no residues at $p_{1}$, it cannot have residues at all. Therefore, it can be integrated globally. Finally, define $\sigma_{12}$ to be one such integral, that is, it satisfies $d \sigma_{12}=\alpha_{2} d \alpha_{1}$.

Proceeding by induction, once $\alpha_{i}, 1 \leq i \leq m$, and $\sigma_{j k}, 1 \leq j<k \leq m$, have been chosen, consider the divisor $N_{m+1} p_{m+1}-D$, where $N_{m}$ is a positive integer. By Riemann-Roch, we have

$$
\operatorname{dim}\left(\mathscr{L}\left(N_{m+1} p_{m+1}-D\right)\right) \geq N_{m+1}-C(D, g),
$$

where $C(D, g)$ denotes a constant depending only on $D$ and $g$.

Consider the linear map

$$
Q_{m+1}: \mathscr{L}\left(N_{m+1} p_{m+1}-D\right) \longrightarrow \overbrace{\mathbb{C}^{2 g} \times \mathbb{C}^{2 g} \times \cdots \times \mathbb{C}^{2 g}}^{m \text { times }}
$$


defined by

$$
f \longrightarrow\left(\int_{\gamma_{1}} f d \alpha_{1}, \ldots, \int_{\gamma_{2 g}} f d \alpha_{1}\right) \times \cdots \times\left(\int_{\gamma_{1}} f d \alpha_{m}, \ldots, \int_{\gamma_{2 g}} f d \alpha_{m}\right) .
$$

Choosing $N_{m+1}$ large enough, we can guarantee that $Q_{m+1}$ has nontrivial kernel. We can then choose $\alpha_{m+1}$ in the kernel of $Q_{m+1}$ so that each form $\alpha_{m+1} d \alpha_{i}(i=1,2, \ldots, m)$ has no residues at $p_{i}$.

Since each of the forms $\alpha_{m+1} d \alpha_{1}, \ldots, \alpha_{m+1} d \alpha_{m}$ has poles only at two points and it does not have residues at one of them, they cannot have residues at all. Thus they can be integrated to yield $\sigma_{1 m+1}, \ldots, \sigma_{m m+1}$, respectively.

Therefore, by induction we can find a solution $\tilde{\Phi}$ of the differential system (2.13). Its degree is at least the polar degree of $\alpha_{1}$, which is $N_{1}$, which was chosen arbitrarily. Thus, the degree of $\tilde{\Phi}$ can be made arbitrarily large.

Also, $\alpha_{2}$ takes different values over the points $\left\{r_{1}, r_{2}, \ldots, r_{N_{1}}\right\}=\alpha_{1}^{-1}(a)$, with a some point in the image of $\alpha_{1}$, so $\tilde{\Phi}$ is generically one to one and it is not a multiple cover of its image.

The only condition that remains to be verified is that $\tilde{\Phi}$ is linearly full. Recall that the map $\tilde{\Phi}$ will be linearly full unless the determinant of $\left(\alpha^{\prime}, \alpha^{\prime \prime}, \ldots, \alpha^{(n)}\right)$ is identically zero. But this cannot happen since by our choices the functions $\alpha_{1}, \alpha_{2}, \ldots, \alpha_{n}$ have poles at different points of $M_{g}$.

\section{Connectedness of the moduli space}

THEOREM 5.1. Given a superminimal map $f \in \operatorname{Supm}_{d}^{n}\left(M_{g}, S^{2 n}\right)$, there is a curve $c(\lambda) \subseteq \operatorname{Supm}_{d}^{n}\left(M_{g}, S^{2 n}\right)$ so that $c(0)=f$ and $c(1) \in \operatorname{Supm}_{d}^{n-1}\left(M_{g}, S^{2 n}\right)$.

REMARK 5.2. This was proved in [10] for $g=0$ using an explicit deformation, and in [8] for higher genus using a deformation in $\mathscr{L}_{n}$. Our proof is similar; we include it for its simplicity and as an example where the techniques developed in the previous sections are used. We use a simple linear deformation in $\mathbb{P}^{N_{n}}$ which induces a deformation in $\mathscr{L}_{n}$.

This theorem is an immediate corollary to the following proposition.

Proposition 5.3. Given $\widetilde{\Phi} \in \mathrm{HH}_{d}^{n}\left(M_{g}, \mathscr{Z}_{n}\right)$, there is a one-parameter family of curves $\widetilde{\Phi}_{\lambda} \subseteq \mathrm{HH}_{d}^{n}\left(M_{g}, \mathscr{L}_{n}\right)(\lambda \in \mathbb{C})$ so that $\widetilde{\Phi}_{1}=\Phi$ and $\widetilde{\Phi}_{0} \in \mathrm{HH}_{d}^{n-1}\left(M_{g}, \mathscr{L}_{n}\right)$.

Proof. The idea is simple: first, use the correspondence $\Phi \leftrightarrow \tilde{\Phi}$ with a proper choice of the base plane so that the degrees of both maps are the same. Then deform $\tilde{\Phi}$ by noting that, for $\lambda \in \mathbb{C}$, the curve defined by

$$
\left[s: \lambda \alpha_{1}: \alpha_{2}: \cdots: \alpha_{n}: \lambda \tau_{12}: \cdots: \lambda \tau_{1 n}: \tau_{23}: \cdots: \tau_{n-1, n}\right]
$$

is also a solution of (2.13). When $\lambda=0$, we have

$$
\left[s: 0: \alpha_{2}: \cdots: \alpha_{n}: 0: \cdots: 0: \tau_{23}: \cdots: \tau_{n-1, n}\right]
$$


This last map is clearly not linearly full, so it corresponds to a map $\Phi_{0}: M_{g} \rightarrow$ $\mathscr{Z}_{n-1}$ as desired.

Of course, either the deformation of $\Phi$ or the deformation of $\tilde{\Phi}$ shown above may not be continuous. However, after a rotation and a few technical points everything works.

If $\Phi \notin \mathrm{HH}_{d}^{f}\left(M_{g}, \mathscr{Z}_{n}\right)$, then there is nothing to prove. Thus we can assume that $\Phi \in \mathrm{HH}_{d}^{f}\left(M_{g}, \mathscr{E}_{n}\right)$. Using Propositions 3.6 and 3.5, choose a base plane $E$ so that $\Phi$ falls in the regular part of $\mathscr{C}_{E}$, and $\tilde{\Phi}=\mathscr{C}_{E}(\Phi)$ satisfies the hypotheses of Proposition 3.3(a). Of course, $\Phi$ and $\widetilde{\Phi}$ have the same degree.

Then $\tilde{\Phi}$ has the property that $\alpha$ is not zero at the points where $s$ vanishes, so we can find a unit vector $v_{2}$ such that $\left(v_{2}, \alpha(p)\right) \neq 0$ at the points $p$ where $s=0$. Let $A \in \operatorname{Gl}(n, \mathbb{C})$ be any matrix with $v_{2}$ as its second row. Then we must have that $A \widetilde{\Phi}$ misses the subspace

$$
\left[0: x_{1}: 0: \cdots: 0: t_{12}: \cdots: t_{1 n}: 0: \cdots: 0\right]
$$

Since the action of $\operatorname{Gl}(n, \mathbb{C})$ is equivariant and continuous in $\mathbb{P}^{N_{n}}$ and $\mathscr{L}_{n}$ (see Lemma 2.6 and Remarks 2.7 and 2.8), $A \widetilde{\Phi}$ has the same degree as $\tilde{\Phi}$, and the problem is reduced to deforming the rotated curve $A \widetilde{\Phi}$. For convenience of notation, we will still use $\tilde{\Phi}$ to denote this rotated curve.

Finally, consider the deformation

$$
\widetilde{\Phi}_{\lambda}:=I(\lambda) \widetilde{\Phi}
$$

where $\lambda \in \mathbb{C}$ and

$$
I(\lambda)=\left(\begin{array}{ccccc}
\lambda & 0 & 0 & \cdots & 0 \\
0 & 1 & 0 & \cdots & 0 \\
0 & 0 & 1 & \cdots & 0 \\
\vdots & \vdots & \vdots & \ddots & \vdots \\
0 & 0 & 0 & \cdots & 1
\end{array}\right)
$$

(This is, we are multiplying by $\lambda$ all the entries $\alpha_{i}, \tau_{j k}$ for which any subindex equals 1.)

This deformation corresponds to a projection $\mathbb{P}^{N_{n}} \rightarrow \mathbb{P}^{N_{n-1}}$ whose center is the subspace given by (5.3). Since $\widetilde{\Phi}$ misses this subspace, this deformation must be continuous for every $\lambda \in \mathbb{C}$. For $\lambda=0$, the entry corresponding to $\alpha_{1}$ is 0 . Hence, $W(\alpha) \equiv 0$, and the curve cannot be linearly full. We have thus found a deformation of $\widetilde{\Phi}$ that satisfies what we want.

The only thing that remains to be checked is that the corresponding deformation of $\Phi$ in $\mathscr{L}_{n}$ is continuous. To this end, observe that the deformation given in (5.4) corresponds to a deformation in $\mathbb{P}^{M_{n}}$ of the form

$$
\left[p_{0}(\lambda) x_{0}: p_{1}(\lambda) x_{1}: \cdots: p_{\ell}(\lambda) x_{\ell}: x_{\ell+1}: x_{\ell+2}: \cdots: x_{M_{n}}\right]
$$


where $p_{i}(\lambda)$ denote positive powers of $\lambda$. Therefore, for $\lambda=0$, it corresponds to a projection $\mathbb{P}^{M_{n}} \rightarrow \mathbb{P}^{M_{n-1}}$.

Thus, the deformation $\tilde{\imath}_{\circ} \tilde{\Phi}_{\lambda}$, is continuous for $\lambda \neq 0$. For $\lambda=0$, it will also be continuous unless the curve $\tilde{\imath} \circ \widetilde{\Phi}$ passes through the center of the projection $\mathbb{P}^{M_{n}} \rightarrow \mathbb{P}^{M_{n-1}}$. But if this was the case, the degree of $\tilde{\imath}_{\circ} \tilde{\Phi}_{0}$ would be less than the degree of $\tilde{\imath}_{\circ} \tilde{\Phi}_{\lambda}, \lambda \neq 0$. Since $\operatorname{deg}\left(\tilde{\imath}_{\circ} \tilde{\Phi}_{0}\right) \geq 2 \operatorname{deg}\left(\tilde{\Phi}_{0}\right)=2 \operatorname{deg}\left(\tilde{\Phi}_{1}\right)=\operatorname{deg}\left(\tilde{\imath}_{\circ}\right.$ $\tilde{\Phi}_{1}$ ), the degree cannot drop at $\lambda=0$, and therefore $\tilde{\imath}_{\circ} \tilde{\Phi}_{\lambda}$ is a continuous deformation for all $\lambda$.

6. The dimension of the moduli space of harmonic spheres in $S^{6}$. Not much is known about the dimension or structure of the moduli space of harmonic maps $S^{2} \rightarrow S^{2 n}$ for $n>2$. For $n=2$, it is known that the dimension of this moduli space is $2 d+4$ (see [11]) and that it is a variety of pure dimension $2 d+4$ with three irreducible components (see [13]). In [1], Barbosa shows that when $d=n(n+1) / 2, \operatorname{Supm}_{d}^{f}\left(S^{2}, S^{2 n}\right)$ is diffeomorphic to $O(2 n+1, \mathbb{C})$.

In the late 1980s, Bolton and Woodward posed the conjecture that the dimension of the moduli space of harmonic maps $S^{2} \rightarrow S^{2 n}$ is $2 d+n^{2}$, which of course coincides with Barbosa's result. An explanation appears in [2], where they also announce a proof of this fact due to Oxbury, but it seems that it was not correct. To our knowledge, there have been no further advances on proving or disproving this conjecture.

In this last section we give a lower bound for the dimension of $\operatorname{Supm}_{d}^{f}\left(S^{2}, S^{6}\right)$ which coincides with the conjectured value. Namely, we show that this dimension is greater than or equal to $2 d+9$. It seems that our method will also work for arbitrary $n$, but we will not develop this here.

Using the results of the previous sections, we have that the top dimension of this moduli space is exactly the top dimension of $\operatorname{PD}_{d}^{f}\left(S^{2}, \mathbb{P}^{N_{3}}\right)$. This dimension is one less (after projectivizing) than the dimension of the set of solutions of the system

$$
\begin{aligned}
& \alpha_{2} d \alpha_{1}-\alpha_{1} d \alpha_{2}=2\left(s d \tau_{12}-\tau_{12} d s\right), \\
& \alpha_{3} d \alpha_{2}-\alpha_{2} d \alpha_{3}=2\left(s d \tau_{23}-\tau_{23} d s\right), \\
& \alpha_{1} d \alpha_{3}-\alpha_{3} d \alpha_{1}=2\left(s d \tau_{31}-\tau_{31} d s\right),
\end{aligned}
$$

subject to the condition

$$
W\left(\left(\frac{\alpha}{s}\right)^{\prime}\right):=\operatorname{det}\left(\begin{array}{lll}
\left(\frac{\alpha_{1}}{s}\right)^{\prime} & \left(\frac{\alpha_{1}}{s}\right)^{\prime \prime} & \left(\frac{\alpha_{1}}{s}\right)^{\prime \prime \prime} \\
\left(\frac{\alpha_{2}}{s}\right)^{\prime} & \left(\frac{\alpha_{2}}{s}\right)^{\prime \prime} & \left(\frac{\alpha_{2}}{s}\right)^{\prime \prime \prime} \\
\left(\frac{\alpha_{3}}{s}\right)^{\prime} & \left(\frac{\alpha_{3}}{s}\right)^{\prime \prime} & \left(\frac{\alpha_{3}}{s}\right)^{\prime \prime \prime}
\end{array}\right) \neq \equiv 0,
$$

where $s, \alpha_{1}, \alpha_{2}, \alpha_{3}, \tau_{12}, \tau_{23}$, and $\tau_{31}$ are polynomials in one complex variable $z$, of degree less than or equal to $d$ with at least one of them of degree $d$, and without a common factor for all of them. 
We will find a $2 d+10$-dimensional set of solutions of (6.1) satisfying (6.2). To this end, we need the following lemma.

LEMMA 6.1. Let $d \geq 8$. Given any polynomial $s(z)$ of degree $d$ with only single zeroes, there exist solutions of (6.1) satisfying (6.2).

Proof. Let $s=\prod_{k=1}^{d}\left(z-s_{k}\right)$. System (6.1) is equivalent to

$$
\alpha_{j} \alpha_{i}^{\prime}-\alpha_{i} \alpha_{j}^{\prime}=2 s^{2}\left(\frac{T_{i j}}{s}\right)^{\prime}, \quad 1 \leq i<j \leq 3
$$

which is equivalent to

$$
\begin{gathered}
\frac{\alpha_{j} \alpha_{i}^{\prime}-\alpha_{i} \alpha_{j}^{\prime}}{s^{2}}, \text { has no residues, } \\
\tau_{i j}=s \int \frac{\alpha_{j} \alpha_{i}^{\prime}-\alpha_{i} \alpha_{j}^{\prime}}{s^{2}} d z \text { is a polynomial of degree } \leq d .
\end{gathered}
$$

Let

$$
\begin{gathered}
E\left(\alpha_{i}, \alpha_{j}\right)\left(s_{m}\right):=\lim _{z \rightarrow s_{m}}\left(\frac{\left(z-s_{m}\right)^{2}\left(\alpha_{j}(z) \alpha_{i}^{\prime}(z)-\alpha_{i}(z) \alpha_{j}^{\prime}(z)\right)}{(s(z))^{2}}\right)^{\prime}, \\
F\left(\alpha_{i}\right)\left(s_{m}\right):=\lim _{z \rightarrow s_{m}}\left[\left(\frac{\left(z-s_{m}\right)^{2}}{(s(z))^{2}}\right)^{\prime} \alpha_{i}^{\prime}(z)+\frac{\left(z-s_{m}\right)^{2}}{(s(z))^{2}} \alpha_{i}^{\prime \prime}(z)\right] .
\end{gathered}
$$

Since $s$ has only simple zeroes, solutions to the system (6.4) satisfy (6.5) implicitly, and for these solutions, system (6.4) is equivalent to the system

$$
E\left(\alpha_{i}, \alpha_{j}\right)\left(s_{m}\right)=0, \quad 1 \leq i<j \leq 3,1 \leq m \leq d-1
$$

(We only need that condition (6.7) be satisfied at $\left\{s_{1}, s_{2}, \ldots, s_{d-1}\right\}$ since the sum of the residues of a meromorphic function is 0 .)

Let $r \geq 3$ with $3 r \leq d+1$. Let $\alpha_{1}$ be a polynomial of degree $d$ or less which is a solution of the system of equations

$$
\begin{aligned}
\alpha_{1}\left(s_{m}\right) & =0, & & 1 \leq m \leq r, \\
F\left(\alpha_{1}\right)\left(s_{m}\right) & =0, & & 1 \leq m \leq r,
\end{aligned}
$$

and such that $\alpha_{1}\left(s_{m}\right) \neq 0$ for $r+1 \leq m \leq d$. (Such an $\alpha_{1}$ can be found explicitly developing (6.8) further and using elementary interpolation; here is where we need that $3 r \leq d+1$.)

Note that, since $\alpha_{1}$ satisfies (6.8), equations (6.7) are implicitly satisfied at the points $\left\{s_{1}, s_{2}, \ldots, s_{r}\right\}$ for $i=1$, and $j=2$ or $j=3$. 
Consider the system of equations

$$
\begin{aligned}
& F\left(\alpha_{2}\right)\left(s_{m}\right)=0, \quad m=1, r+1, \\
& F\left(\alpha_{3}\right)\left(s_{m}\right)=0, \quad m=2, r+1, \\
& E\left(\alpha_{2}, \alpha_{3}\right)\left(s_{m}\right)=0, \quad 3 \leq m \leq r, \\
& E\left(\alpha_{1}, \alpha_{2}\right)\left(s_{m}\right)=0, \quad r+2 \leq m \leq d-1, \\
& E\left(\alpha_{3}, \alpha_{1}\right)\left(s_{m}\right)=0, \quad r+2 \leq m \leq d-1,
\end{aligned}
$$

where $\alpha_{2}$ and $\alpha_{3}$ are polynomials of degree $d$ or less. Note that $\alpha_{2}=s$ and $\alpha_{3}=s$ are a solution of this system.

We have $4+4+(r-2)+2(d-r-2)=2 d+2-r$ equations and $2 d+2$ unknowns. Thus, the solution set has dimension at least $r \geq 3$. Therefore, we can find $\alpha_{2}$ and $\alpha_{3}$ so that the set $\left\{s, \alpha_{2}, \alpha_{3}\right\}$ is linearly independent and such that $\alpha_{2}\left(s_{2}\right) \neq 0$ and $\alpha_{3}\left(s_{1}\right) \neq 0$.

Take $\alpha_{2}$ and $\alpha_{3}$ as specified in the last paragraph. Using the relation

$$
\alpha_{1}\left(s_{m}\right) E\left(\alpha_{2}, \alpha_{3}\right)\left(s_{m}\right)+\alpha_{2}\left(s_{m}\right) E\left(\alpha_{3}, \alpha_{1}\right)\left(s_{m}\right)+\alpha_{3}\left(s_{m}\right) E\left(\alpha_{1}, \alpha_{2}\right)\left(s_{m}\right)=0,
$$

it is easy to see that $\left(s, \alpha_{1}, \alpha_{2}, \alpha_{3}\right)$ is a solution of system (6.7), and hence of (6.1). Also, by construction, these polynomials have no common factors.

It remains to check that they satisfy condition (6.1). This is immediate since $\alpha_{1} / s$ has a pole at $s_{r+1}$ while $\alpha_{2} / s$ and $\alpha_{3} / s$ do not, $\alpha_{2} / s$ has a pole at $s_{2}$ while $\alpha_{1} / s$ and $\alpha_{3} / s$ do not, and $\alpha_{3} / s$ has a pole at $s_{1}$ while $\alpha_{1} / s$ and $\alpha_{2} / s$ do not.

THEOREM 6.2. For $d \geq 6, d \neq 7$, the dimension of the moduli spaces of linearly full harmonic maps $S^{2} \rightarrow S^{6}$ of degree $d$ is at least $2 d+9$.

Proof. For $d=6$, Barbosa's result in [1] gives $\operatorname{dim}\left(\operatorname{Supm}_{d}^{f}\left(S^{2}, S^{2 n}\right)\right)=2 d+9$.

Define the Zariski open subset of $\mathbb{C}^{4 d+4}$ given by

$$
\mathscr{S}=\left\{\left(s, \alpha_{1}, \alpha_{2}, \alpha_{3}\right) \in\left(\mathbb{C}[z]_{d}\right)^{4}: s \text { has } d \text { distinct roots }\right\}
$$

Let

$$
\begin{aligned}
\mathscr{R}=\{ & \left(s, \alpha_{1}, \alpha_{2}, \alpha_{3}\right) \in \mathscr{S}: W\left(\left(\frac{\alpha}{s}\right)^{\prime}\right) \neq 0, E\left(\alpha_{i}, \alpha_{j}\right)\left(s_{m}\right)=0, \\
& \left.1 \leq i<j \leq 3,1 \leq m \leq d-1, \text { where }\left\{s_{1}, s_{2}, \ldots, s_{d}\right\} \text { are the roots of } s\right\} .
\end{aligned}
$$


Using relation (6.10), we see that system (6.7) has no more than $2 d-3$ independent homogeneous equations and $4 d+4$ variables. (It is not immediate that the number of independent equations is no more than $2 d-3$ as it may seem that the correct bound is $2 d-2$. To see that $2 d-3$ is the correct bound, one has to play with the equations a bit. We omit the details.) The set $\mathscr{R}$ is the intersection of the variety given by (6.7) with the open set given by condition (6.2). By Lemma $6.1, \mathscr{R}$ is not empty. Therefore its dimension is at least $2 d+7$.

Finally, define

$$
\begin{gathered}
\mathscr{A}=\left\{\left(s, \alpha_{1}, \alpha_{2}, \alpha_{3}, \tau_{12}, \tau_{23}, \tau_{31}\right) \in\left(\mathbb{C}[z]_{d}\right)^{7}:\left(s, \alpha_{1}, \alpha_{2}, \alpha_{3}\right) \in \mathscr{R},\right. \\
\left.\tau_{i j}=\frac{1}{2}\left(a_{i j} s+s \int \frac{\alpha_{j} \alpha_{i}^{\prime}-\alpha_{i} \alpha_{j}^{\prime}}{s^{2}} d z\right), 1 \leq i<j \leq 3\right\},
\end{gathered}
$$

where $a_{i j}$ are arbitrary complex numbers (integration constants).

The elements of $\mathscr{A}$ are solutions of (6.1) satisfying (6.2). The dimension of $\mathscr{A}$ is the dimension of $\mathscr{R}$ plus the three extra degrees of freedom given by the integration constants. Thus, $\operatorname{dim}(\mathscr{A}) \geq 2 d+10$. Therefore we have $\mathbb{P} \mathscr{A} \subset$ $\operatorname{PD}_{d}^{f}\left(S^{2}, \mathbb{P}^{N_{3}}\right)$ and $\operatorname{dim}(\mathbb{P} \mathscr{A}) \geq 2 d+9$. This proves the theorem.

AcKNowledgment. I would like to thank my thesis adviser, Professor QuoShin Chi, for all his support and guidance.

\section{REFERENCES}

[1] J. L. M. Barbosa, On minimal immersions of $S^{2}$ into $S^{2 m}$, Trans. Amer. Math. Soc. 210 (1975), 75-106.

[2] J. Bolton and L. M. Woodward, Moduli spaces of harmonic 2-spheres, Geometry and Topology of Submanifolds, IV (Leuven, 1991) (F. Dillen and L. Verstraelen, eds.), World Scientific, New Jersey, 1992, pp. 143-151.

[3] R. L. Bryant, Conformal and minimal immersions of compact surfaces into the 4-sphere, J. Differential Geom. 17 (1982), no. 3, 455-473.

[4] _ Submanifolds and special structures on the octonians, J. Differential Geom. 17 (1982), no. 2, 185-232.

[5] _ Lie groups and twistor spaces, Duke Math. J. 52 (1985), no. 1, 223-261.

[6] E. Calabi, Minimal immersions of surfaces in Euclidean spheres, J. Differential Geometry 1 (1967), 111-125.

[7] M. Furuta, M. A. Guest, M. Kotani, and Y. Ohnita, On the fundamental group of the space of harmonic 2-spheres in the n-sphere, Math. Z. 215 (1994), no. 4, 503-518.

[8] M. A. Guest and Y. Ohnita, Group actions and deformations for harmonic maps, J. Math. Soc. Japan 45 (1993), no. 4, 671-704.

[9] J.-I Hano, Conformal immersions of compact Riemann surfaces into the $2 n$-sphere $(n \geq 2)$, Nagoya Math. J. 141 (1996), 79-105.

[10] M. Kotani, Connectedness of the space of minimal 2-spheres in $S^{2 m}(1)$, Proc. Amer. Math. Soc. 120 (1994), no. 3, 803-810.

[11] B. Loo, The space of harmonic maps of $S^{2}$ into $S^{4}$, Trans. Amer. Math. Soc. 313 (1989), no. 1, 81-102.

[12] M.-L. Michelsohn, Surfaces minimales dans les sphères [Minimal surfaces in spheres], Astérisque (1987), no. 154-155, 115-130 (French). 
[13] J.-L. Verdier, Two dimensional $\sigma$-models and harmonic maps from $S^{2}$ to $S^{2 n}$, Group Theoretical Methods in Physics, (Proc. 11th int. Colloq., Istanbul 1982), Lecture Notes in Physics, vol. 180, Springer-Verlag, Berlin, 1983, pp. 136-141.

[14] - Applications harmoniques de $S^{2}$ dans $S^{4}$ [Harmonic mappings from $S^{2}$ into $S^{4}$ ], Geometry Today (Rome, 1984) (E. Arbarello, C. Procesi, and E. Strickland, eds.), Progr. Math., vol. 60, Birkhäuser Boston, Massachusetts, 1985, pp. 267-282 (French).

[15] _ Applications harmoniques de $S^{2}$ dans $S^{4}$. II [Harmonic mappings from $S^{2}$ into $S^{4}$. II], Harmonic Mappings, Twistors, and $\sigma$-Models (Luminy, 1986) (P. Gauduchon, ed.), Adv. Ser. Math. Phys., vol. 4, World Scientific, Singapore, 1988, pp. 124-147 (French).

Luis Fernández: Departamento de Matemáticas, Universidad de los Andes, Apartado Aereo 4976, Bogotá, Colombia

E-mail address: 1 fernand@uni andes. edu.co 


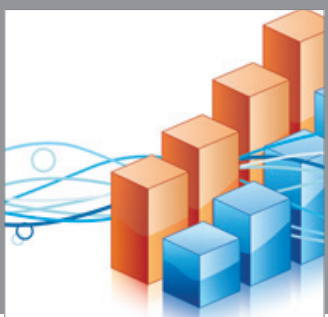

Advances in

Operations Research

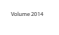

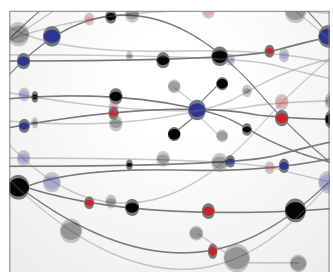

\section{The Scientific} World Journal
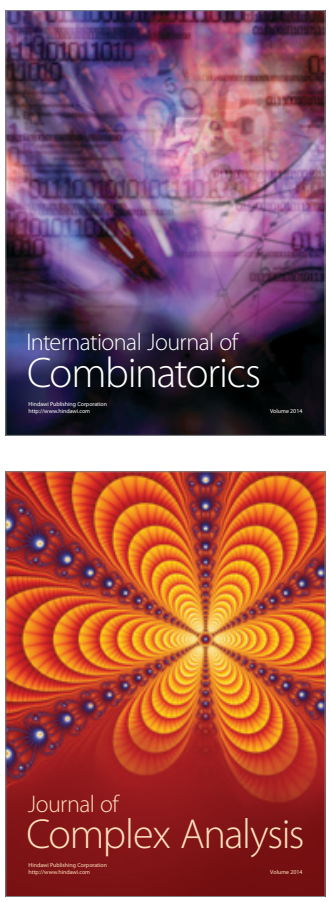

International Journal of

Mathematics and

Mathematical

Sciences
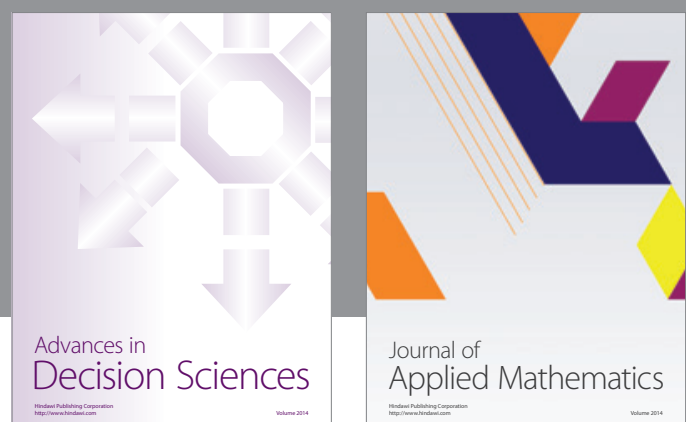

Journal of

Applied Mathematics
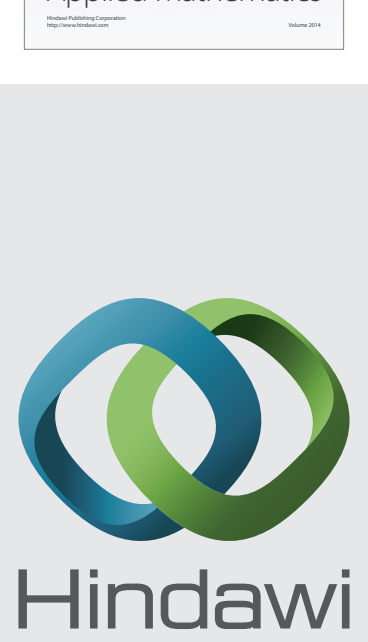

Submit your manuscripts at http://www.hindawi.com
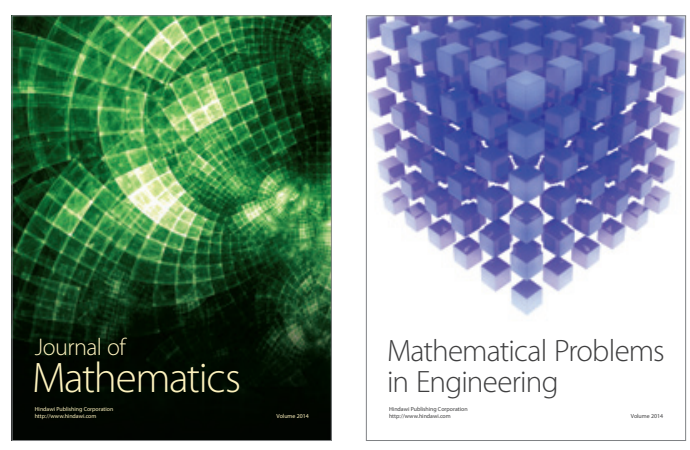

Mathematical Problems in Engineering
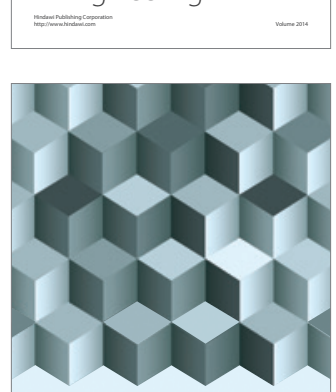

Journal of

Function Spaces
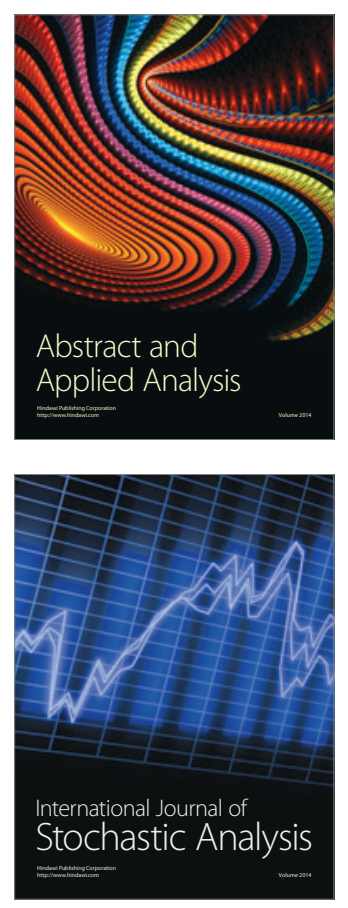

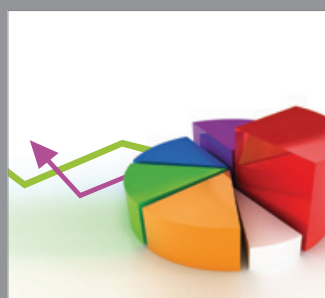

ournal of

Probability and Statistics

Promensencen
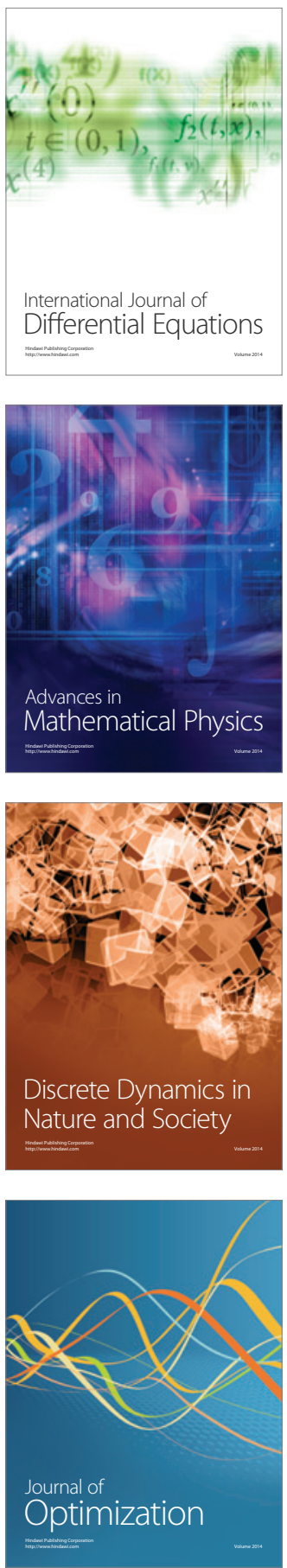